\title{
Combined cryotherapy and external beam radiation therapy for the treatment of intermediate-risk localized prostate cancer: A case series
}

\author{
Peter $\mathbf{Q i}^{1}$, Matvey Tsivian $^{1}$, Zeljko Vujaskovic ${ }^{2}$, Thomas J. Polascik ${ }^{1^{*}}$ \\ ${ }^{1}$ Division of Urology, Department of Surgery, Duke University Medical Center, Durham, USA; \\ *Corresponding Author: thomas.polascik@duke.edu \\ ${ }^{2}$ Department of Radiation Oncology, Duke University Medical Center, Durham, USA
}

Received 10 November 2013; revised 8 December 2013; accepted 31 December 2013

Copyright (C 2014 Peter Qi et al. This is an open access article distributed under the Creative Commons Attribution License, which permits unrestricted use, distribution, and reproduction in any medium, provided the original work is properly cited. In accordance of the Creative Commons Attribution License all Copyrights (C) 2014 are reserved for SCIRP and the owner of the intellectual property Peter Qi et al. All Copyright (C) 2014 are guarded by law and by SCIRP as a guardian.

\section{ABSTRACT}

Introduction: Routine PSA screening for prostate cancer $(\mathrm{PCa})$ has increased the detection of intermediate-risk, localized disease. Conventional treatments for localized PCa include surgery, brachytherapy, cryotherapy or external beam radiotherapy (EBRT). However, for intermediate risk patients, rates of recurrence are moderately high and a multi-modal treatment approach for these patients may be necessary. We treated patients with a combination of cryotherapy and low dose EBRT to assess the safety and feasibility of this combinatory approach as well as to evaluate early oncological outcomes. Case Presentation: Men with intermediate risk (PSA $=10-20 \mathrm{ng} / \mathrm{ml}$ and/or Gleason $=7$ and/or clinical T2b) localized PCa were prospectively enrolled in this study. Patients underwent cryotherapy and then 39 Gy EBRT 4 - 6 weeks after surgery. After completing EBRT, the men were followed every 3 months for 2 years. Adverse events, PSA, urinary and erectile function were assessed during each follow-up. Three patients completed the study. Preoperative PSA ranged from 3.5 to $7.9 \mathrm{ng} / \mathrm{ml}$. There were no intraoperative complications and the treatment was well tolerated. Following cryotherapy and EBRT, all patients were pad-free within 6 months and remained continent for the duration of the study. Bother index remained stable throughout the study for all patients. No urethral strictures or rectal toxicities were observed. PSA remained undetectable for all patients. Conclusions: In this prospective study, cryotherapy combined with low dose EBRT was a safe approach for the treatment of intermediate-risk, localized PCa. Early oncological outcomes appeared to be favorable with all patients having undetectable PSA during the 2-year follow-up period. Further studies are warranted to confirm these preliminary results.

\section{KEYWORDS}

\section{External Beam Radiation; Cryotherapy; Localized Prostate Cancer; Intermediate Risk}

\section{INTRODUCTION}

Routine PSA screening for prostate cancer (PCa) has increased the detection of localized disease, which can be divided into three risk groups: low, intermediate, and high [1,2]. Patients presenting these tumors may be treated with a variety of interventions including radical prostatectomy, cryotherapy, external beam radiotherapy (EBRT), and brachytherapy [3-5]; however, biochemical failure for patients with intermediate-risk disease is documented in approximately $20 \%$ - $40 \%$ of cases [6-8]. An improvement in the cure rate is needed for these patients. The American Urological Association (AUA) clinical guidelines have suggested a multi-faceted treatment approach for localized PCa and encourage patients with localized disease to enroll in clinical trials investigating combinatorial treatment strategies [9]. In recent years, cryotherapy has gained ground as a viable alternative for treating localized PCa [10-15]. Compared to the conventional surgery, cryotherapy is less invasive and 
has a shorter recovery period $[16,17]$. Recurrence following cryotherapy is likely due to failure in the periprostatic (extracapsular) tissue although failure may also occur either in the central zone surrounding the urethra due to the protective effect of the warming catheter or the prostatic apex [18]. On the other hand, the treatment failure following EBRT is likely to arise from the prostate as a result of restricting the necessary amount of radiation required to eliminate the tumor while minimizing damage to surrounding tissues. Given that cryotherapy and radiation may be complementary in that each may account for the potential deficiencies of one another, it is of great interest to assess the efficacy of a combination cryotherapy-EBRT treatment for localized PCa.

We conducted the first step of a phase I dose-escalation study combining cryotherapy with EBRT. The aim of this trial was to assess the safety and feasibility of performing cryotherapy with subsequent radiation therapy and assess early oncological outcomes of this approach. In this study, cryotherapy took the place of brachytherapy as a local treatment for intermediate-risk PCa.

\section{CASE PRESENTATION}

After approval from the Institutional Review Board, adult men who had biopsy confirmed, localized PCa with intermediate risk [2] (PSA 10 - 20 ng/ml and/or Gleason score of 7 and/or clinical T2b) with prostate volume $\leq 60$ cc were prospectively enrolled in this phase I study between 2007-2009 at this institution. All patients had a negative metastatic work up consisting of a bone scan. Additional staging imaging studies (CT, MR) were ordered at the physician's discretion. The patients underwent a complete history and physical exam that assessed sexual function, urinary function, bowel function, medical comorbidities, a rectal examination, and PSA level. Exclusion criteria included Karnofsky performance status (KPS) $<90 \%$, prior pelvic radiation therapy, chemotherapy, or androgen deprivation therapy (ADT).

This study was designed to consist of 4 levels of doseescalating EBRT. We intended to include 24 patients with 6 patients in each level; however due to poor accrual, enrollment was closed prematurely. The Common Toxicity Criteria for Adverse Events (CTCAE) v3.0 criteria was used to grade complications and assess doseescalating criteria.

Patients were scheduled to undergo whole gland cryotherapy followed by EBRT 4 - 6 weeks after surgery. Our cryotherapy technique has been described elsewhere [14]. Briefly, we utilized third generation cryotechnology using 17G probes (Galil Medical Arden Hills, Minnesota) with transrectal ultrasound guidance. Cryoneedles were placed in standard fashion with each needle localized in both transverse and sagittal dimensions. Two freeze-thaw cycles were performed using Argon +/- helium gas, respectively; optimal freezing temperature was gauged to be $<-20^{\circ} \mathrm{C}$. At least 2 thermoprobes were placed, one each at the urinary sphincter and the anterior rectal surface. Patients were discharged with a temporary urinary catheter and returned to clinic within 2 weeks.

Radiation was to be administered in a dose-escalation fashion, starting at 39.6 Gy for a maximum of 54 Gy, within a $2 \mathrm{~cm}$ margin including the prostate and seminal vesicles, except posteriorly where the margin was 0.7 $1.0 \mathrm{~cm}$. A planning CT scan and/or MRI were also obtained to contour all relevant structures. Intensitymodulated radiotherapy (IMRT) or 3-dimensional conformal planning was employed to enable maximal blocking of relevant tissues and to ensure full coverage of the prostate inferiorly. Prescription dose was delivered to the highest isodose line that completely covered the target volume. For patients in group 1, the prostate and seminal vesicles would receive 39.6 Gy at $1.8 \mathrm{~Gy}$ per fraction. Patients in group 2 would receive an additional 5.4 Gy boost to the same volume, for a total of $45.0 \mathrm{~Gy}$. In group 3 the final boost dose would be $50.4 \mathrm{~Gy}$, and in group 4 it would be $54 \mathrm{~Gy}$.

Follow-up consisted of a post-cryotherapy (prior to EBRT) and post-EBRT visit, and every 3 months thereafter for 2 years after completion of radiation treatment. During each visit, adverse events (urinary incontinence, stricture, and rectal toxicity etc.) were assessed according to CTCAE v3.0 criteria; quality of life and urinary bother index were evaluated using the CTCAE and the American Urological Association Symptom Score questionnaire (International Prostate Symptom Score and prostate Bother Index combined), as well as sexual function (the International Index of Erectile Function, IIEF-5). Sexual potency was also evaluated on a scale of 1 to 5 ( $1=$ able to have unassisted intercourse and $5=$ unable to have erections on own/not interested in trying interventions). Urinary continence was also assessed by patient reported daily pad usage. Biochemical failure was defined as nadir $+1 \mathrm{ng} / \mathrm{mL}$ or PSA $>0.5 \mathrm{ng} / \mathrm{mL}$ after treatment.

\section{RESULTS}

A total of 6 patients were enrolled in this prospective study. However, only 3 patients completed the study protocol. One patient withdrew prior to cryosurgery; another chose to undergo EBRT with ADT; a third patient developed a urinary tract infection post-cryotherapy and opted not to proceed on the study.

Table 1 details disease characteristics and patient demographics. All patients had biopsy Gleason scores of 7. Clinical stage at presentation ranged from $\mathrm{T} 1 \mathrm{c}$ to $\mathrm{T} 2 \mathrm{a}$ disease; baseline PSA levels ranged from 3.5 to 7.9 $\mathrm{ng} / \mathrm{mL}$, percent-free PSA from 0.5\% - 15.2\%, and IIEF-5 
Table 1. Patient demographics and disease characteristics.

\begin{tabular}{|c|c|c|c|c|c|c|c|}
\hline $\begin{array}{l}\text { Patient } \\
\text { No. }\end{array}$ & Race & $\begin{array}{c}\text { Age } \\
\text { (Years) }\end{array}$ & $\begin{array}{l}\text { Gleason } \\
\text { Score }\end{array}$ & $\begin{array}{c}\text { Clinical } \\
\text { Stage }\end{array}$ & $\begin{array}{l}\text { PSA } \\
\text { NG/ML }\end{array}$ & $\begin{array}{c}\text { \% Free } \\
\text { PSA }\end{array}$ & IIEF-5 \\
\hline 1 & Cauc & 74 & $7(4$ & T2a & 3.5 & 11.6 & 1 \\
\hline 2 & Cauca & 72 & $7(3$ & $\mathrm{T} 2 \mathrm{a}$ & 7.9 & 15.2 & 1 \\
\hline 3 & Caucasian & 66 & $7(3+4)$ & T1c & 4.0 & 0.5 & 7 \\
\hline
\end{tabular}

PSA = Prostate specific antigen (Pre-treatment); IIEF-5 = International Index of Erectile Function.

scores from 1 - 7. All 3 patients received the same level of radiation (39.6 Gy) during EBRT.

There were no intraoperative complications and the immediate post-cryotherapy course was uneventful for these 3 patients. On the post-cryotherapy follow-up visit, patients reported using 1 - 3 pads. All patients were padfree within 6 months following cryotherapy-EBRT and remained continent for the duration of the study. Table 2 details patients' functional outcomes. Of note, bother index remained stable at baseline levels throughout the duration of the study for all patients, whereas IPSS decreased in 1 , remained stable in 1 and increased in 1 from baseline to the conclusion of the study. No additional adverse events as defined by the CTCAE v3.0 occurred. Specifically, there were no instances of urethral stricture, slough, or rectal toxicity in this series. All patients had undetectable PSA $(<0.2 \mathrm{ng} / \mathrm{ml})$ after 2 years of follow-up. No patients required additional therapy.

\section{DISCUSSION}

In this study, we report on the outcomes of cryoablation with EBRT as a combinatorial treatment modality for intermediate risk prostate cancer. This is the first study of its kind to assess a combination cryotherapyEBRT intervention for localized, intermediate-risk PCa. We found cryoablation and low dose EBRT was feasible and safe in these 3 patients, demonstrating promising short-term outcomes whereby all patients had undetectable PSA levels during the 2 year follow-up.

Conventional treatment of intermediate risk localized PCa includes brachytherapy, EBRT, cryotherapy, and surgery (i.e. radical prostatectomy [RP]) [4]; however, failure rates for these patients are estimated to be $20 \%$ $40 \%$ [6-8]. The reasons for failure vary with the type of intervention. For instance, recurrence after RP is likely to arise from positive surgical margins and extracapsular extension [7,19-21]. Due to the nature of radiation emitted from the implanted seed and rapid dose fall in the periprostatic regions, failure from brachytherapy is likely to arise from the surrounding tissues [3]. Biochemical failure after cryotherapy is likely to occur in the periprostatic tissues, although failure has also been documented in the seminal vesicles [18]. In contrast, EBRT
Table 2. Urinary and erectile function in patients undergoing cryoablation and external beam radiation for localized prostate cancer.

\begin{tabular}{ccccccc}
\hline & & \multicolumn{5}{c}{ Follow-up visit } \\
\hline \multirow{2}{*}{ IPSS } & Patient & Preoperative & 3 mos & 9 mos & 12 mos & 24 mos \\
& 1 & 4 & 5 & 11 & 15 & 15 \\
& 2 & 22 & 2 & 10 & 20 & 15 \\
Bother index & 1 & 2 & 2 & 2 & 2 & 2 \\
& 3 & 6 & 3 & 6 & 5 & 5 \\
& 2 & 4 & 0 & 3 & 4 & 3 \\
& 3 & 0 & 0 & 0 & 0 & 0 \\
& 1 & 1 & 1 & 1 & 1 & 1 \\
& 2 & 1 & 1 & 1 & 1 & 1 \\
& 3 & 7 & 1 & 2 & 1 & 18 \\
\hline
\end{tabular}

IPSS = International Prostate Symptom Score; IIEF-5 = International Index of Erectile Function.

delivers radiation to both the prostate and periprostatic regions and is potentially effective at eliminating microscopic disease surrounding the prostate [22]. However, radiation delivered may be limited in dose to minimize the damage to surrounding tissues, possibly causing failure from EBRT to arise primarily from within the prostate. As a result of the competing potential failures presented by these two treatment modalities, we proposed a cryotherapy-EBRT combination approach to treating intermediate risk localized PCa. The study was designed such that cryotherapy preceded EBRT in order to optimize the therapeutic effects of freezing before the bowel/rectum is irradiated. If EBRT is the primary treatment, then a less aggressive freeze would have to be employed in order to protect the tissues in these areas.

Cryotherapy combined with low dose EBRT proved to be both a feasible and safe treatment modality in this study. Common side effects of cryotherapy or EBRT alone include urinary incontinence, urinary stricture, impotence, and/or rectal toxicity $[10,23]$. Therefore, a primary concern of combining the two treatment techniques was the exacerbation of these adverse effects. However, we did not observe any urinary stricture, rectal toxicity during this series. All patients became continent within 6 months and remained so for the duration of the study. It is important to note that one patient had documented detrusor instability prior to enrollment and was documented to have used urinary pads for approximately 2 months following cryotherapy. Finally, it should be noted that all 3 patients maintained undetectable PSA levels during the 2 year follow-up period.

A major limitation to this study is the very small sample size. In fact, the study was closed prematurely due to 
poor accrual which, among many reasons, was due to the time commitments of EBRT. Because Duke University Medical Center is a tertiary referral institution, many of our patients were not local and the inconvenience associated with transportation to and from Duke for daily radiation over several-week periods deterred many eligible patients from participating. As a result of our small patient cohort, we were unable to assess the results of escalating radiation doses up to $54 \mathrm{~Gy}$ as per protocol and therefore we are unable to suggest whether there may be an optimal radiation dose in this setting. Furthermore, despite encouraging short-term cancer control outcomes, long-term oncological results require further maturity.

\section{CONCLUSION}

This study is the first report assessing combined cryotherapy-EBRT treatment for localized intermediate risk PCa. In this prospective study, cryotherapy combined with low dose EBRT was a safe approach for the treatment of localized, intermediate risk prostate cancer. Furthermore, short-term oncological control for these patients was promising. These initial results are intriguing and require confirmation in future studies.

\section{REFERENCES}

[1] Littrup, P.J. (1997) Future benefits and cost-effectiveness of prostate carcinoma screening. American Cancer Society. Cancer, 80, 1864-1870.

http://dx.doi.org/10.1002/(SICI)1097-0142(19971101)80: 9<1864::AID-CNCR27>3.0.CO;2-2

[2] D’Amico, A.V., Whittington, R., Malkowicz, S.B., et al. (1998) Biochemical outcome after radical prostatectomy, external beam radiation therapy, or interstitial radiation therapy for clinically localized prostate cancer. The Journal of the American Medical Association, 280, 969-974. http://dx.doi.org/10.1001/jama.280.11.969

[3] Nag, S., Beyer, D., Friedland, J., Grimm, P. and Nath, R. (1999) American Brachytherapy Society (ABS) recommendations for transperineal permanent brachytherapy of prostate cancer. International Journal of Radiation Oncology*Biology*Physics, 44, 789-799.

http://dx.doi.org/10.1016/S0360-3016(99)00069-3

[4] Tewari, A. (2003) Management of clinically localized prostate cancer: Pathologic processing to robotic prostatectomy. Reviews in Urology, 5, S33-S39.

[5] Sharkey, J., Cantor, A., Solc, Z., et al. (2002) Brachytherapy versus radical prostatectomy in patients with clinically localized prostate cancer. Current Urology Reports, 3, 250-257. http://dx.doi.org/10.1007/BF03200421

[6] Quaranta, B.P., Marks, L.B. and Anscher, M.S. (2004) Comparing radical prostatectomy and brachytherapy for localized prostate cancer. Oncology (Williston Park), 18, 1289-1302; Discussion 302-304, 309.

[7] Kupelian, P.A., Elshaikh, M., Reddy, C.A., Zippe, C. and
Klein, E.A. (2002) Comparison of the efficacy of local therapies for localized prostate cancer in the prostatespecific antigen era: A large single-institution experience with radical prostatectomy and external-beam radiotherapy. Journal of Clinical Oncology, 20, 3376-3385. http://dx.doi.org/10.1200/JCO.2002.01.150

[8] Vicini, F.A., Martinez, A., Hanks, G., et al. (2002) An interinstitutional and interspecialty comparison of treatment outcome data for patients with prostate carcinoma based on predefined prognostic categories and minimum follow-up. Cancer, 95, 2126-2135.

http://dx.doi.org/10.1002/cncr.10919

[9] Thompson, I., Thrasher, J.B., Aus, G., et al. (2007) Guideline for the management of clinically localized prostate cancer: 2007 update. Journal of Urology, 177, 21062131. http://dx.doi.org/10.1016/j.juro.2007.03.003

[10] Caso, J.R., Tsivian, M., Mouraviev, V., Kimura, M. and Polascik, T.J. (2011) Complications and postoperative events after cryosurgery for prostate cancer. BJU International, 109, 840-845.

[11] Donnelly, B.J., Saliken, J.C., Ernst, D.S., et al. (2002) Prospective trial of cryosurgical ablation of the prostate: Five-year results. Urology, 60, 645-649. http://dx.doi.org/10.1016/S0090-4295(02)01839-3

[12] Robinson, J.W., Donnelly, B.J., Saliken, J.C., Weber, B.A., Ernst, S. and Rewcastle, J.C. (2002) Quality of life and sexuality of men with prostate cancer 3 years after cryosurgery. Urology, 60, 12-18. http://dx.doi.org/10.1016/S0090-4295(02)01679-5

[13] Han, K.R., Cohen, J.K., Miller, R.J., et al. (2003) Treatment of organ confined prostate cancer with third generation cryosurgery: Preliminary multicenter experience. Journal of Urology, 170, 1126-1130. http://dx.doi.org/10.1097/01.ju.0000087860.52991.a8

[14] Polascik, T.J., Nosnik, I., Mayes, J.M. and Mouraviev, V. (2007) Short-term cancer control after primary cryosurgical ablation for clinically localized prostate cancer using third-generation cryotechnology. Urology, 70, 117121. http://dx.doi.org/10.1016/j.urology.2007.03.027

[15] Babaian, R.J., Donnelly, B., Bahn, D., et al. (1993) Best practice statement on cryosurgery for the treatment of localized prostate cancer. Journal of Urology, 180, 19932004. http://dx.doi.org/10.1016/j.juro.2008.07.108

[16] Ahmed, S. and Davies, J. (2005) Managing the complications of prostate cryosurgery. BJU International, 95, 480481. http://dx.doi.org/10.1111/j.1464-410X.2005.05379.x

[17] Han, K.R. and Belldegrun, A.S. (2004) Third-generation cryosurgery for primary and recurrent prostate cancer. BJU International, 93, 14-18. http://dx.doi.org/10.1111/j.1464-410X.2004.04547.x

[18] Shinohara, K., Rhee, B., Presti Jr., J.C. and Carroll, P.R. (1997) Cryosurgical ablation of prostate cancer: Patterns of cancer recurrence. Journal of Urology, 158, 2206-2209; Discussion 9-10. http://dx.doi.org/10.1016/S0022-5347(01)68198-9

[19] Bolla, M., Collette, L., Blank, L., et al. (2002) Long-term results with immediate androgen suppression and external irradiation in patients with locally advanced prostate can- 
cer (an EORTC study): A phase III randomised trial. Lancet, 360, 103-106.

http://dx.doi.org/10.1016/S0140-6736(02)09408-4

[20] Leibovich, B.C., Engen, D.E., Patterson, D.E., et al. (2000) Benefit of adjuvant radiation therapy for localized prostate cancer with a positive surgical margin. Journal of Urology, 163, 1178-1182. http://dx.doi.org/10.1016/S0022-5347(05)67717-8

[21] Carson 3rd, C.C., Zincke, H., Utz, D.C., Cupps, R.E. and Farrow, G.M. (1980) Radical prostatectomy after radiotherapy for prostatic cancer. Journal of Urology, 124,
237-239.

[22] Anscher, M.S., Robertson, C.N. and Prosnitz, R. (1995) Adjuvant radiotherapy for pathologic stage T3/4 adenocarcinoma of the prostate: Ten-year update. International Journal of Radiation Oncology*Biology*Physics, 33 37-43. http://dx.doi.org/10.1016/0360-3016(95)00038-Z

[23] Odrazka, K., Dolezel, M., Vanasek, J., et al. (2010) Time course of late rectal toxicity after radiation therapy for prostate cancer. Prostate Cancer and Prostatic Diseases, 13, 138-143. http://dx.doi.org/10.1038/pcan.2009.56 